\title{
Milankovitch forcing in paleoclimate data
}

\author{
István Matyasovszky* \\ Department of Meteorology, Eötvös Loránd University, Pázmány Péter sétány 1/A, 1117 Budapest, Hungary
}

\begin{abstract}
This study addresses the strength of the statistical relationship between paleoclimate data and the Earth orbital forcing. A methodology called the nonparametric additive model (NPAM) was used to relate $\delta^{18} \mathrm{O}$ data of the North Greenland Ice Core Project (NGRIP) ice core and reconstructed temperatures of the Vostok ice core to eccentricity, obliquity, and precession. Both data sets depended linearly on precession and approximately linearly on obliquity, but relations to eccentricity had more complex forms. Percentage variance reductions obtained by these 3 Earth orbital parameters were almost identical for the 122950 yr NGRIP data and the 422766 yr Vostok data. Specifically, variance explained by eccentricity was $40.2 \pm 3 \%$ and $36.6 \pm 1.7 \%$ of the variance of NGRIP and Vostok data, respectively. The contributions of obliquity and precession to the variance were $27.9 \pm 3 \%$ and $31.1 \pm 1.7 \%$ for the 2 data sets.
\end{abstract}

KEY WORDS: Earth orbital parameters · Milankovitch cycles · NGRIP $\cdot$ North Greenland Ice Core Project $\cdot$ Vostok $\cdot$ Nonparametric regression

\section{INTRODUCTION}

Long-term climate variations are dominated by cycles essentially identical to the cycles of Earth orbital parameters. According to the theory of Milankovitch, variations of eccentricity, obliquity, and longitude of perihelion (precession) modify the spatio-temporal quantity of solar influx resulting in climate variations. A first convincing support for Milankovitch's theory (Hays et al. 1976) identified 3 discrete spectral peaks in ocean-floor sediment data at periods of approximately 100000,42000 , and $23000 \mathrm{yr}$ that roughly correspond to the periods of Earth orbital parameters.

As Milankovitch cycles have been found in numerous data sets, a question to be addressed is how strong is the statistical relationship between paleoclimate data and Earth orbital forcing. A spectral analysis by Wunsch (2004) performed with 100000 yr Vostok core deuterium data reported only 7 to $10 \%$ of the total variance caused by periods corresponding to obliquity and precession. The role of eccentricity was not clear due to the record length. Combining a careful evaluation of Vostok ice core data with advanced spectral analysis techniques, Meyers et al. (2008) showed a $41 \%$ contri- bution of cycles corresponding to eccentricity and a $28 \%$ contribution of obliquity and precession to the total variance for data from the last 423000 yr. Although Meyers et al. (2008) convincingly criticized the approach of Wunsch (2004), the large difference between their results warrants further examination. Note that these studies used only paleoclimate data and did not take orbital parameters into account.

Here I relate paleoclimate data to Earth orbital parameters using eccentricity, obliquity, and the cosine and sine of the longitude of perihelion. As the climate response to astronomical forcing is possibly nonlinear, paleoclimate data were regressed on these predictors with no assumption on the analytical form of the relationship using a multivariate nonparametric regression technique. At this step of the procedure, a climate response does not uniquely connect to a given value of the forcing but depends on whether this forcing is in its increasing or decreasing stages (Imbrie \& Imbrie 1980). Therefore, 4 additional predictors, namely derivatives of the 4 original predictors, were also included in the analysis (Liu 1995). Dependence on the derivatives is likely attributable to the response time of the climate system (Imbrie \& Imbrie 
1980, Roe 2006). Derivatives are approximated by differences between 2 consecutive values of the 4 orbital parameters and are then divided by the angular frequencies related to their leading periodicities. Note, however, that this division has no role; its purpose is simply to avoid working with values that are too small. The methodology was applied to oxygen isotope values of the North Greenland Ice Core Project $\left(\delta^{18} \mathrm{O}\right.$ of NGRIP) core and reconstructed temperatures of the Vostok Ice Core.

\section{DATA AND METHODOLOGY}

\subsection{Data}

The relative difference $\delta^{18} \mathrm{O}$ between a given ratio ${ }^{18} \mathrm{O} /{ }^{16} \mathrm{O}$ of water and the ratio ${ }^{18} \mathrm{O} /{ }^{16} \mathrm{O}$ of Standard Mean Ocean Water is widely used as a proxy for climate because $\delta^{18} \mathrm{O}$ depends nearly linearly on temperature (Jouzel et al. 1994). Hence higher $\delta^{18} \mathrm{O}$ values indicate higher temperatures at the time that snow falls. $\delta^{18} \mathrm{O}$ NGRIP (2004) data are available for the last $122950 \mathrm{yr}$ as $50 \mathrm{yr}$ averages. The core was cross-dated to the GRIP core ss09sea chronology (Johnsen et al. 2001) for the last 105000 yr. Older ice was cross-dated to the Antarctic Vostok ice core records by using concentrations of methane and $\delta^{18} \mathrm{O}$. Reconstructed Vostok temperature is available for the last $422766 \mathrm{yr}$ as the temperature difference with respect to the present value. The time scale was GT4 (Petit et al. 1999), which has previously been used (Wunsch 2004, Meyers et al. 2008). Due to the growing compaction of deeper layers of the ice, the resulting data are unevenly spaced in time, ranging from 17 to 664 yr. High-accuracy orbital parameter data calculated after Berger \& Loutre (1992) were obtained from http://aom.giss.nasa.gov/srorbpar.html for each year considered in the analysis.

\subsection{Nonparametric regression}

Several versions of nonparametric regressions have no assumptions on the analytical form of these regression surfaces. The final form of a nonparametric regression technique consists of moving local linear smoothing of data representing the variable to be estimated (predictand). The locality, called bandwidth, is defined in the predictor space as a neighborhood of actual predictor variables, and the predictand values involved in the smoothing are accompanied by predictors lying in this neighbourhood. Coefficients for smoothing are generated by a suitable kernel function. Note that bandwidths play a crucial role in the accu- racy of the procedure. Large bandwidths, allowing large amounts of smoothing, deliver small variances with possibly large biases, while small bandwidths provide large variances with small biases. Thus, an optimal bandwidth recognizing the trade-off between the bias and variance must be estimated. Numerous mathematical papers have discussed these techniques; here I refer the reader to Simonoff (1996).

Direct estimation of a multivariate regression surface is limited by the 'curse of dimensionality'. The curse of dimensionality is due to the fact that representation of a surface requires drastically increasing the number of data with increasing dimension of the surface. This study involved an 8-dimensional case, as the predictors used in the analysis include eccentricity, obliquity, and cosine and sine of the longitude of perihelion, as well as derivatives of these 4 variables. A way around this difficulty is to use additive models arriving at 1-dimensional nonparametric regressions. In a 1-dimensional case, local linear smoothing using weighted local regression (WLR) has advantageous properties (Fan 1992, 1993, Fan \& Gijbels 1992). Let $y_{1}, y_{2}, \ldots, y_{n}$ be a time series representing consecutive paleoclimate data, and let $\mathbf{x}(1)=\left[x_{1}(1), \ldots, x_{p}(1)\right]^{T}, \ldots, \mathbf{x}(n)=\left[x_{1}(n), \ldots, x_{p}(n)\right]^{T}$ be a vector time series of $p$ predictors, where $T$ denotes transpose. The nonparametric additive model (NPAM) is defined as

$$
m[\mathbf{x}(i)]=E\left[y_{i} \mid \mathbf{x}(i)\right]=\sum_{j=1}^{p} m_{j}\left[x_{j}(i)\right]
$$

where $m_{j}\left[x_{j}(i)\right]$ represents a particular estimate for $y_{i}$ with $x_{j}(i)$ and $E$ refers to the expected value operation. The additive model Eq. (1) is estimated by the following iterative procedure. First, for $j=1, \ldots, p$ the WLR estimate assumes that $m_{j}\left(x_{j}\right)$ is smooth for any $x_{j}$ satisfying $\min _{i}\left[x_{j}(i)\right] \leq x_{j} \leq \min _{i}\left[x_{j}(i)\right]$, and hence it is approximated linearly in a neighborhood of $x_{j}$ as $m_{j}\left[x_{j}(i)\right]$ $\approx \alpha_{j}(i)+\beta_{j}(i)\left[x_{j}(i)-x_{j}\right]$. The quantity

$$
\sum_{i=1}^{n}\left(y_{i}-\left\{\alpha_{j}+\beta_{j}\left[x_{j}(i)-x_{j}\right]\right\}\right)^{2} K\left[\frac{x_{j}(i)-x_{j}}{h}\right]
$$

is minimized with respect to $\alpha_{j}$ and $\beta_{j}$, and $\hat{m}\left(x_{j}\right)=\hat{\alpha}$. The kernel function is the Epanechnikov kernel (Fan 1992), defined as $K(u)=3 / 4\left(1-u^{2}\right)$ in $[-1,1]$ and 0 otherwise, while $h$ is the bandwidth. In the second step, $z_{i}=y_{i}-\sum_{j \neq k} \hat{m}_{j}\left[x_{j}(i)\right]$ is formed, and the procedure of the first step is applied with the substitution of $y_{i}$ by $z_{i}$ for every $k$. This step is repeated until estimates $\hat{m}_{j}\left(x_{j}\right)$ stabilize (Chen \& Tsay 1993). There are several techniques to estimate bandwidths in nonparametric problems; to save space, the details are omitted here (Francisco-Fernandez \& Vilar-Fernandez 2004). Note that small bandwidths indicate highly nonlinear relationships, while large (infinite) bandwidths show approximately (global) linear relationships. 


\section{APPLICATION}

First, a linear regression (LR) of Vostok data on orbital parameters was performed. This step delivered a residual variance of 6.462 and 5.006, reducing the variance of 8.356 by $22.7 \%$ and $40.1 \%$ for the 4 -variate (omitting the derivatives of orbital parameters) and the 8-variate (including the derivatives) case, respectively. The NPAM procedure provided variance reductions of $42.3 \%$ (without derivatives) and $67.7 \%$ (including derivatives). A comparison of these 4 percentages demonstrates both the nonlinear climate response to orbital forcing and the importance of derivatives of orbital elements. Fig. 1 clearly shows a substantially better performance of NPAM over LR. However, the NPAM curve at approximately 350000 yr ago (and before) fits the data relatively poorly. This is related to the behavior of eccentricity and its derivative. Near this time, the eccentricity (its derivative) reaches its minimum (maximum) observed during the period examined. Hence, a relatively small amount of reconstructed temperatures are smoothed here when calculating the NPAM estimate for an actual eccentricity, because only few data are accompanied by eccentricity data lying in the bandwidth-defined neighborhood of the actual eccentricity. In order to demonstrate the climate response to Milankovitch forcing, the estimate of the reconstructed temperature obtained with NPMA is illustrated for the predictor pair of obliquity (Fig. 2). In the case of linearity, the points should be placed on a plane; this condition is almost satisfied for obliquity where bandwidths are large. However, the predictor pair of eccentricity accompanied by small estimated bandwidths exhibits

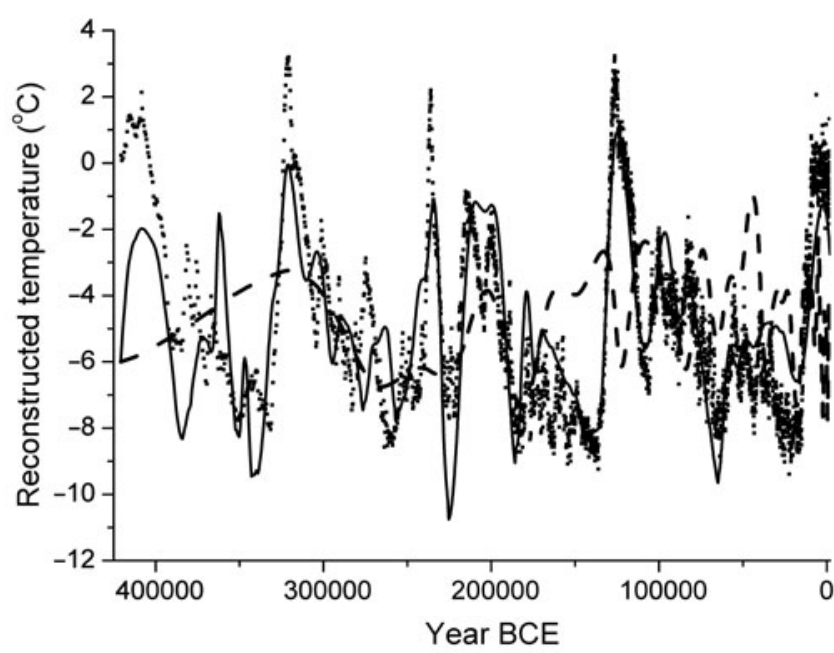

Fig. 1. Reconstructed temperatures from the Vostok ice core and their estimates obtained by nonparametric additive (solid) and linear regression (dashed) models for the last $422766 \mathrm{yr}$ using Earth orbital parameters. Temperatures are differences with respect to the present value. BCE: before Common Era a much more complex form (Fig. 3a,b). To obtain a clearer picture on the climate response to eccentricity, calculations were repeated with 10-fold enlarged bandwidths. Fig. 3c,d, exhibiting a smoother curve, with attached loops according to the 4 cycles of around
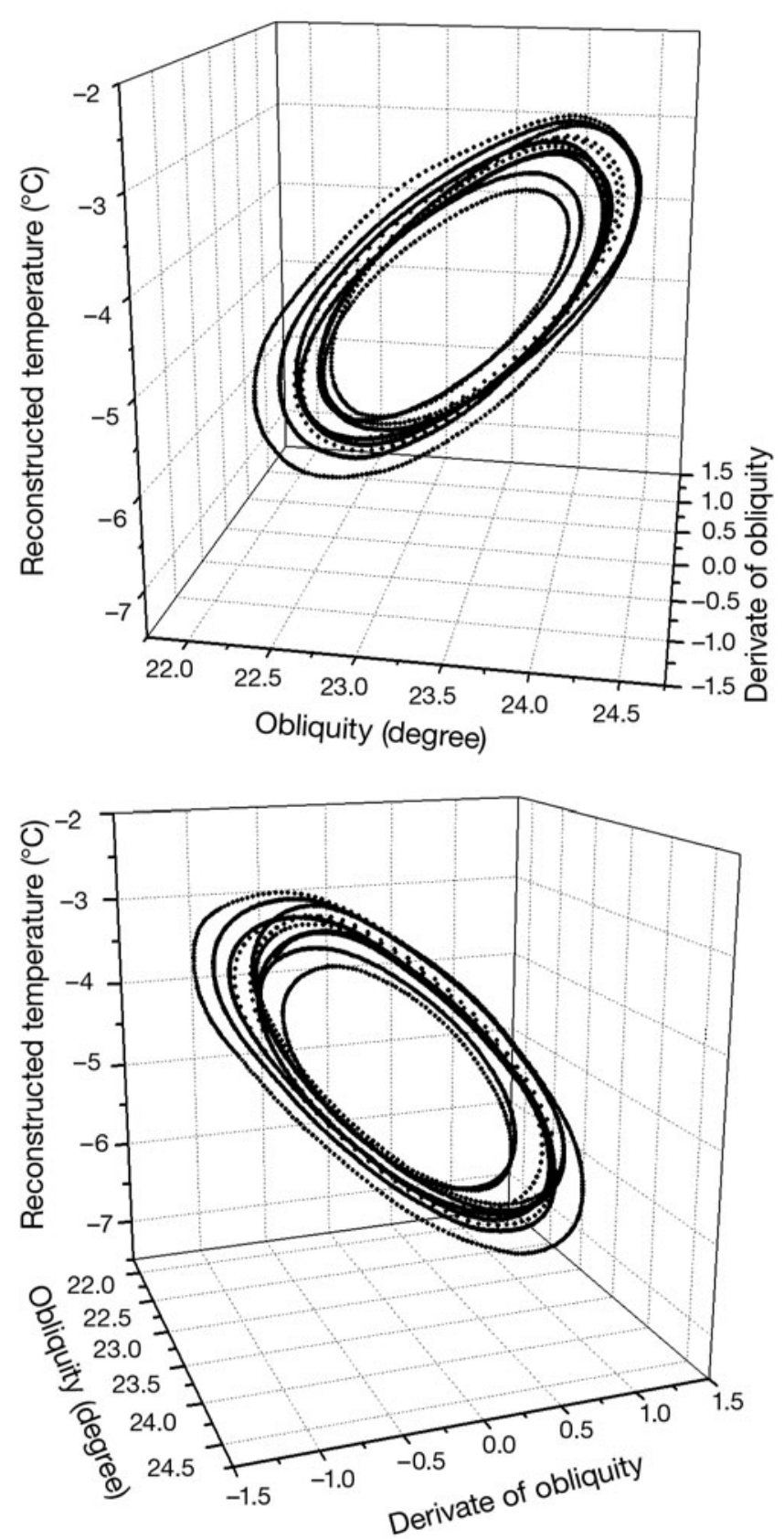

Fig. 2. Relation of reconstructed temperatures from the Vostok ice core on obliquity for the last $422766 \mathrm{yr}$. Temperatures are differences with respect to the present value. The derivative of obliquity was approximated by differences between 2 consecutive values of obliquity divided by the angular frequency corresponding to a $100000 \mathrm{yr}$ period. Its unit is therefore identical to the unit of obliquity. The upper and lower panels show the same graph, but at different angles 

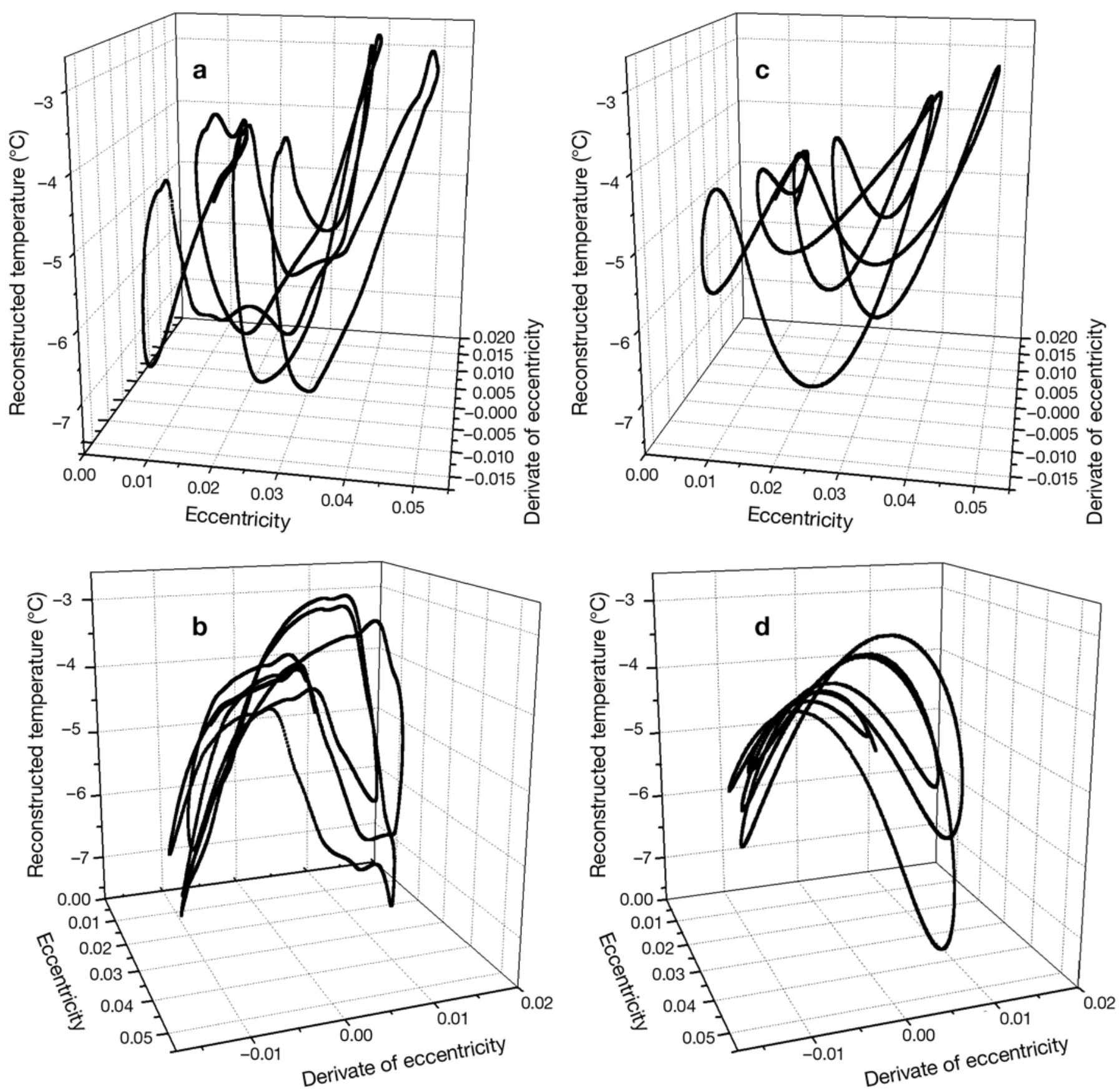

Fig. 3. Relation of reconstructed temperatures from the Vostok ice core on eccentricity for the last 422766 yr obtained with (a,b) optimal bandwidths and (c,d) tenfold enlarged bandwidths. Temperatures are differences with respect to the present value. The derivative of eccentricity was approximated by differences between 2 consecutive values of eccentricity divided by the angular frequency corresponding to a $100000 \mathrm{yr}$ period. The upper and lower panels show the same graphs, but at different angles

100000 yr during the period examined. However, Fig. 3c,d is included simply for illustration, as the bandwidths used here were chosen arbitrarily, whereas Fig. 3a,b was produced with the estimated optimal bandwidths. Fig. 3a,b shows more clearly that the most rapid climate changes appear when the deriv- ative of eccentricity approaches its maximum and minimum values (Liu 1995). The relationship of precession to the data is linear (infinite bandwidths) and relatively weak, albeit statistically significant. This latter can be shown with the NPAM fitting procedure (Chen \& Tsay 1993). Also, the need for derivatives in the model was 
checked according to Chen \& Tsay (1993). The rank of importance of the 3 orbital parameters in view of their explained variances is as follows: eccentricity, obliquity, and precession. This can be shown by partial correlations between paleoclimate data and the additive terms in Eq. (1) corresponding to the 3 orbital parameters. An important task is to quantify particular contributions of these 3 elements to the total explained variance. However, the members in Eq. (1) may correlate and thus the sum of individual contributions of the cycles to the variance may be larger than the variance explained together by these cycles. When $v_{1}, v_{2}$, and $v$ denote the variance explained by eccentricity, obliquity, and precession together and all of the 3 orbital elements, respectively, the individual contribution of eccentricity is between $v_{1}-\left(v_{1}+v_{2}-v\right)=v-v_{2}$ and $v_{1}$. Similarly, the individual contribution of obliquity and precession is at least $v-v_{1}$ and at most $v_{2}$. Hence, the eccentricity explains 34.9 to $38.3 \%$ of the total variance, while obliquity and precession together account for 29.4 to $32.8 \%$ of the variance.

An LR of $\delta^{18} \mathrm{O}$ NGRIP data on orbital parameters delivers a residual variance of 4.801 and 3.944, reducing the variance 8.667 by $44.6 \%$ and $54.5 \%$ for the 4 -variate (omitting the derivatives of orbital parameters) and the 8-variate (including the derivatives) cases, respectively. The NPAM procedure provides variance reductions of $49.5 \%$ without derivatives and $68.1 \%$ including derivatives. These percentages show that the climate response to orbital forcing is more direct here than for Vostok because the relationship is better approximated linearly and including derivatives carries smaller gains. Note that the percentage $68.1 \%$ is essentially identical to the percentage obtained for the Vostok data. Also, the rank of the 3 individual orbital parameters for NGRIP is the same as in the previous case. The eccentricity explains 37.2 to $43.2 \%$ of the total variance, while obliquity and precession together account for 24.9 to $30.9 \%$ of the variance. These values are close to those obtained for Vostok, but eccentricity plays a slightly larger role, while obliquity and precession play somewhat lesser roles compared to the Vostok data.

\section{DISCUSSION AND CONCLUSIONS}

A number of research papers have demonstrated that climate is linearly forced by obliquity and precession cycles, whereas the 100000 yr cycle in paleoclimate data is due to the nonlinear variability arising internally within the climate system (Imbrie et al. 1993, Raymo 1997, Tziperman et al. 2006). Findings of my analysis are in agreement with these facts, as both data sets depended linearly on precession and approxi- mately linearly on obliquity, whereas relations to eccentricity had more complex forms. My methodology showed that $40.2 \pm 3 \%$ and a $27.9 \pm 3 \%$ of the variance in the NGRIP data were explained by variations in eccentricity and in obliquity and precession. An application of my approach to Vostok data revealed that eccentricity contributed $36.6 \pm 1.7 \%$ to the variance and obliquity and precession contributed $31.1 \pm 1.7 \%$.

To better compare results obtained for NGRIP and Vostok, I also applied this method to the last 122975 years of Vostok-reconstructed temperatures. As a result, the amount of variance explained was $40.9 \pm$ $17.5 \%$ for eccentricity and $33.3 \pm 17.5 \%$ for obliquity and precession, providing a total variance reduction of $74.2 \%$. This latter percentage is markedly higher than the value of $67.7 \%$ obtained for the complete data set. This is likely because the temporal resolution of data decreases into the past, and thus sparser data accompanied by sparser orbital element values produce less accurate estimations when going back in time (see Fig. 1). The contribution of obliquity and precession seems more stable in time than that of eccentricity, strengthening the idea that the 100000 yr cycle in paleoclimate data is developed principally by nonlinear interactions within the climate system. Note, however, that distinguishing between the roles of different orbital parameters is fairly uncertain in the shortened data set, because redundancy among variances explained by orbital parameters is much larger for the last 122975 yr than for the entire period. Hence, no differences between the 2 periods can be detected.

Comparison of my findings to those of Meyers et al. (2008) requires some caution, because Meyers et al. (2008) did not include variations of orbital parameters in their methodology; they used only Vostok ice core data. My study shows a slightly smaller role of eccentricity, with greater importance of obliquity and precession. My value of $31.1 \pm 1.7 \%$ for obliquity and precession is not likely to be overestimated, as Vostok data depend linearly on precession and nearly linearly on obliquity due to the large bandwidths obtained (infinite for precession). As smaller variance reductions can be achieved with larger bandwidths, an experiment with infinite bandwidth for both obliquity and precession was performed, resulting in a negligible decrease of the explained variance. Similarly, my value of $36.6 \pm 1.7 \%$ for eccentricity is unlikely to be underestimated, as small bandwidths ( 0.012 for eccentricity and 0.003 for its derivative) recognizing finer structures of relationships were used for this orbital element.

Time delays between $\delta^{18} \mathrm{O}$ or reconstructed temperature and orbital forcing were involved in the NPAM for 2 reasons: (1) it directly considers the response time of the climate system, and (2) it takes into account the reconstructed time errors (age model uncertainty). The 
joint effect of the response time and the average of reconstructed time errors were expected to be identified with such delays. Several delays in a wide range in both time directions were selected, but the optimal statistical relationships delivering the largest variance reductions appeared at the 0 delay. As the average of time-scale errors is likely not 0 (Mudelsee et al. 2009), the effects of the response time and average scale error are likely well balanced. Another possibility is that the average time-scale error is 0 and the response time is taken into account via derivatives of the orbital elements. Evaluating the effects of predefined timescale error models on results is highly complicated (Mudelsee et al. 2009) and is beyond the scope of this study. I selected the time scales used here to allow for a comparison of my results to those of Meyers et al. (2008).

\section{LITERATURE CITED}

Berger A, Loutre MF (1992) Astronomical solutions for paleoclimate studies over the last 3 million years. Earth Planet Sci Lett 111:369-382

Chen R, Tsay R (1993) Nonlinear additive ARX models. J Am Stat Assoc 88:955-967

Fan J (1992) Design-adaptive nonparametric regression. J Am Stat Assoc 87:998-1004

Fan J (1993) Local linear regression smoothers and their ninimax efficiency. Ann Math Stat 21:196-216

Fan J, Gijbels I (1992) Variable bandwidth and local linear regression smoothers. Ann Math Stat 20:2008-2036

Francisco-Fernandez M, Vilar-Fernandez J (2004) Weighted local nonparametric regression with dependent errors: study of real private residential fixed investment in the USA. Stat Infer Stoch Process 7:69-93

Hayes JD, Imbrie J, Shackleton NJ (1976) Variations in the

Editorial responsibility: Mikhail Semenov,

Harpenden, UK
Earth's orbit pacemaker of the ice ages. Science 194: 1121-1132

Imbrie J, Imbrie JZ (1980) Modeling the climatic response to orbital variations. Science 207:943-953

Imbrie J, Berger A, Boyle EA, Clemens SC and others (1993) On the structure and origin of major glaciation cycles. 2 . The 100 000-year cycle. Paleoceanography 8:699-735

Johnsen SJ, Dahl-Jensen D, Gundestrup N, Steffensen JP and others (2001) Oxygen isotope and palaeotemperature records from six Greenland ice-core stations: Camp Century, Dye- 3, GRIP, GISP2, Renland and NorthGRIP. J Quat Sci 16:299-307

Jouzel J, Koster RD, Suozzo RJ, Russell GL (1994) Stable water isotope behavior during the last glacial maximum: a general circulation model analysis. J Geophys Res 99:25791-25801

- Liu HS (1995) A new view on the driving mechanism of Milankovitch glaciation cycles. Earth Planet Sci Lett 131: $17-26$

Meyers S, Saegman BB, Pagani M (2008) Resolving Milankovitch: consideration of signal and noise. Am J Sci 308: 770-786

Mudelsee M, Scholz D, Röthlisberger R, Fleitmann D, Mangini A, Wolff EW (2009) Climate spectrum estimation in the presence of timescale errors. Nonlinear Process Geophys $16: 43-56$

Petit JR, Jouzel J, Raynaud D, Barkov NI and others (1999) Climate and atmospheric history of the past 420000 years from the Vostok Ice Core, Antarctica. Nature 399:429-436

Raymo ME (1997) The timing of major climate terminations. Paleoceanography 12:577-585

Roe G (2006) In defense of Milankovitch. Geophys Res Lett 33:L24703. doi:10.1029/2006GL027817

Simonoff JS (1996) Smoothing methods in statistics. Springer, New York

Tziperman E, Raymo ME, Huybers P, Wunsch W (2006) Consequences of pacing the Pleistocene 100-kyr ice ages by nonlinear phase locking to Milankovitch forcing. Paleoceanography 21:PA4206. doi:1029/2005PA001241

> Wunsch C (2004) Quantitative estimate of the Milankovitchforced contribution to observed Quaternary climate change. Quat Sci Rev 23:1001-1012

Submitted: October 20, 2009; Accepted: February 9, 2010 Proofs received from author(s): March 25, 2010 